quality of life, trial outcome index and total scores all exceeding the minimum clinically important difference. No improvement was seen with standard care.

Conclusions Data from this pilot study suggest intravenous iron improves quality of life, ferritin and transferrin saturations. It may also increase or maintain haemoglobin, thus preventing transfusions after the first cycle of chemotherapy. However, larger adequately powered studies are required to definitively conclude this.

\section{PWE-120 AN EXPERIENCE OF HOME TOTAL PARENTERAL NUTRITION AT THE FREEMAN HOSPITAL OVER A 21- YEAR PERIOD}

Satyasheel Ramful* ${ }^{*}$ Hayley Leyland, Colette Louise Kirk, Lisa Gemmell, Christopher G Mountford, Nicholas P Thompson. Depts of Gastroenterology and Dietetics, Freeman Hospital, Newcastle Upon Tyne, UK

\subsection{6/gutjnl-2018-BSGAbstracts.354}

Introduction Home total parenteral nutrition (HPN) is used for patients with severe intestinal failure. Our service at the Freeman Hospital, Newcastle upon Tyne covers Northumberland, North Cumbria, Durham, Gateshead, North Yorkshire, Tyneside, Wearside and Teesside.

Methods This study used the database kept by the HPN service and the Trust document portal to gather data retrospectively regarding 221 patients started on HPN from 1996 till 2016.25 individual data sets were collated about each patient. Line sepsis rates were obtained from the trust central venous catheter surveillance data (this includes data for patient episodes at other hospitals).

Results The study showed that over the 21 years, there has been a rapid growth in number of patients on HPN.

Number of patients on HPN by year.

The gender split of the cohort was 101 males and 120 females, with a total mean age 52.7 years and median age 55 years. Teesside $(22 \%, 49 / 221)$ followed by Northumberland $(18 \%, 40 / 221)$ were the regions with the most patients initiated on HPN.

The three most common indications for HPN were Postoperative complications (30.3\%, 67/221), Crohn's disease (21.3\%, 47/221) and Mesenteric ischaemia (16.7\%, 37/221). $14.9 \%(33 / 221)$ patients were on $\mathrm{HPN}$ as a result of malignancy.

The 1 year and 5 year survival for the cohort was 94\% and $79.4 \%$ respectively. There were 73 deaths noted in the study group, 8 patients had stopped HPN prior to death. 29 of these patients had been on HPN of a result of malignancy. Of the 65 patients who died while being on HPN, only four deaths were clearly as a complication of parenteral nutrition. 8 patients had neuroendocrine tumours; their average time on HPN was significantly greater at 25.6 months compared to 5.6 months for patients with other tumour types.

The mean rate of catheter-related bloodstream infection was 0.27 per 1000 catheter days over the period covering years 2011 till 2017. The rate of liver (significant fibrosis/cirrhosis) or biliary complications while being on HPN was 12\% (27/221) and the commonest complication was biliary obstruction/cholangitis $(33.3 \%, 9 / 27) .6$ patients of the cohort underwent successful small bowel transplantation and were able to stop HPN.

Conclusions The HPN service at the Freeman has noted a rapid growth in patient numbers, with survival rates comparable to or better than that reported in the literature. Mortality was linked to disease process rather than HPN complications and line infection rate was low.

\section{PWE-121 NUTRITIONAL ASSESSMENT AND VITAMIN DEFICIENCIES IN PATIENTS WITH NETS}

${ }^{1}$ Mark Reynolds*, ${ }^{2}$ Sheryl Lim, ${ }^{3}$ Aled Rees, ${ }^{2}$ Reyhan Chaudhry, ${ }^{1}$ Jennifer Blackhouse, ${ }^{1}$ Mohid S Khan. 'Department of Gastroenterology, Cardiff and Vale University Health Board, Cardiff, UK; ${ }^{2}$ Cardiff University, Cardiff, UK; ${ }^{3}$ Department of Endocrinology, Cardiff and Vale University Health Board, Cardiff, UK

\subsection{6/gutjnl-2018-BSGAbstracts.355}

Introduction Neuroendocrine tumours (NETs) have diverse natural history and clinical syndromes. As a result of the disease or related to management, patients may have altered gut or pancreatic function that can cause nutritional deficiencies. There is a lack of consistent evidence-based dietetic guidance for patients with NETs.

This study evaluated whether nutritional status and nutritional deficiencies had been assessed in patients with NETs in an existing service in South Wales.

Methods A retrospective study included 141 NET patients seen in Gastroenterology $(n=74)$ and Endocrinology $(n=67)$ clinics. Key parameters collected were: BMI, weight, vitamin B12, Ferritin, Folate, Albumin, vitamins $\mathrm{A} / \mathrm{D} / \mathrm{E}$ and presence of steatorrhoea. Evidence of treatment with vitamin or iron replacement and use of bile acid sequestrants or Creon was also recorded.

Results Weight was recorded in under half of patients $(70 / 141)$ and BMI in just $14 \%(n=20)$. This rose to $100 \%$ and $73 \%$ respectively in patients seen by a gastro-specialist dietitian; only 22 patients $(16 \%)$ had this specialist input. 54 patients reported weight loss, $70 \%$ of these $(n=38)$ had a quantified weight loss, $46 \%$ had percentage weight loss calculated.

106 patients $(75 \%)$ had been investigated for a form of vitamin or iron deficiency. The likelihood of investigation was significantly higher in Gastroenterology clinic patients than Endocrine clinics (95\% vs. $54 \%, \mathrm{p}<0.01$ ). $57 \%$ of those investigated were found to have a deficiency, which was consistent across specialities: 59\% $(41 / 70)$ of those from Gastroenterology clinics and 53\% (19/36) from Endocrine clinics. 41/60 patients (68\%) with a recorded deficiency did not have sufficient replacement. $7 / 27$ patients with iron deficiency were given supplementation. 38 patients had vitamin D levels tested (27\%), 29 were insufficient (76\%).

27 patients reported steatorrheoa, 26 of whom were prescribed somatostatin analogues. $96 \%$ of these patients were also prescribed Creon.

Conclusions Although higher rates of nutritional assessment were found in patients who had been assessed by Gastroenterology and with gastro-specialist dietetic involvement, assessment and management of nutritional status in patients with NETs remains an unmet need. Further evidence is required to evaluate nutritional assessment in NETs.

\section{PWE-122 NON-COELIAC GLUTEN/FRUCTAN SENSITIVITY OR IRRITABLE BOWEL SYNDROME (IBS)?}

${ }^{1}$ Kamran Rostami, ${ }^{1}$ Jafer Ali, ${ }^{2}$ Alison Parr, ${ }^{1}$ Ravi Madhotra, ${ }^{1}$ George Macfaul, ${ }^{3}$ Mohammad RostamiNejad. 'Department of Gastroenterology, Milton Keynes University Hospital, Milton Keynes, UK; ${ }^{2}$ Free Lance Nutrition Therapist, Manchester, UK; ${ }^{3}$ Gastroenterology and Liver Diseases Research Institute Shahid Beheshti University of Medical Sciences, Tehran, Iran

10.1136/gutjnl-2018-BSGAbstracts.356 
Introduction A large number of unexplained gastro-intestinal disorders have been attributed to Irritable Bowel Syndrome (IBS) for decades. Discovery of food sensitivities and high rate of symptomatic response to low FODMAP diet has revolutionised the treatment of these conditions. The aim of this study was to assess the triggering factors and the effect of dietary intervention in relation with body mass index (BMI).

Methods This was a cross-sectional study evaluating patients presenting with IBS symptoms referred to our secondary and tertiary Gastroenterology outpatients during January 2014 and July 2016. The total number included in this study were 149 patients. The patients were selected and included based on Salerno expert criteria. A strict gluten and lactose free diet (G/LFD) was recommended for 6 weeks. Out of 149 patients, 134 completed the study and were assessed after following a G/LFD and gluten challenge afterward. Demographics, presenting symptoms, serologic and histological data were recorded.

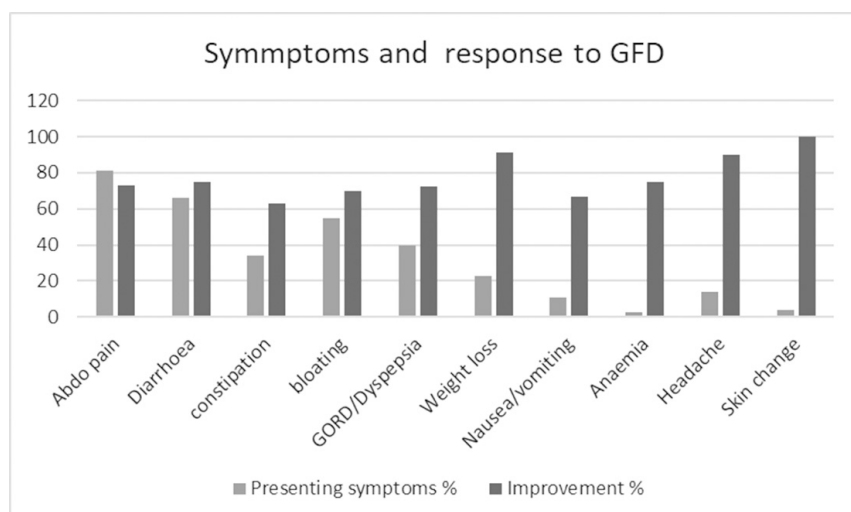

Abstract PWE-122 Figure 1 Prevalence of symptoms shown by percentage and their response to GFD. Following G/LFD introduction $72.4 \%$ (97/134 cases) improved with a score between 30\%-100\%. Abdominal pain was the most prevalent symptoms affecting $80 \%$ of these patients and anaemia the least prevalent presentation affecting $3 \%$ of the study group. The percentage of Improvement scores are shown by brown column

Results The ages of 134 study subjects ranged from 8 to 85 years, with mean age of 46.41 years and standard deviation of 17.388. Patients were predominantly female 109 (81.34\%) in comparison to male 25 (18.66\%). The presenting gastrointestinal symptoms and the rate of their improvement are shown in figure 1 . The majority of this group had an improvement rate close to $100 \%$. Around $53 \%$ of the study population became nearly completely asymptomatic, while $27.6 \%$ had a poor response (scoring $<30 \%$ ) to G/LGD. The improvement score was excellent in patients with normal BMI and good in overweight patients. However a BMI above 30 or below 18 was associated with a poorer response. Over $50 \%$ of these patients didn't require any further follow-up within 12 months.

Conclusion Although it is unclear whether these symptoms are triggered by gluten, fructans, or the other grains components, the elimination of gluten containing grains improved the life quality of these patients with high satisfaction. With exception in patients with higher BMI $>30$ or below 18 , the dietary intervention, was effective and has reduced the number of outpatient visits.

\section{PWE-123 PROSPECTIVE STUDY OF A LOW FODMAP DIET IN PATIENTS WITH COELIAC DISEASE AND IBS SYMPTOMS}

Nick Trott*, Matthew Kurien, David Sanders. Department of Gastroenterology Royal Hallamshire Hospital, Sheffield, UK

\subsection{6/gutjnl-2018-BSGAbstracts.357}

Introduction Patients with coeliac disease (CD) symptoms usually respond to a gluten free diet (GFD). However up to a quarter of adults with $\mathrm{CD}$ have persistent gastrointestinal (GI) symptoms despite strict adherence to a GFD and complete intestinal healing. Overlap between CD and irritable bowel syndrome (IBS) may explain this symptomology. Over the last decade there has been a renewed interest in dietary treatments in IBS particularly the low FODMAP diet (LFD) which temporally restricts fermentable carbohydrates. The aim of this prospective study was to investigate the efficacy of the LFD in patients with CD and IBS. Methods We conducted a prospective study of the LFD as an intervention in patients with treated-CD and persisting IBS symptoms. All CD patients met the ROME IV criteria, had negative coeliac serology and normal duodenal biopsy. All patients were reviewed by a specialist GI dietitian with experience in delivering the LFD. Symptom response was assessed using the validated Gastrointestinal Symptom Rating Scale (GSRS) from base-line to follow up.

Results 24 patients with a mean age of 44 years $(S D=15.2)$ met the inclusion criteria. 8 patients chose not to pursue treatment and 2 were unable to complete the minimum treatment period. 14 patients ( 2 males) completed four weeks of a LFD. There were no differences in baseline demographics between patients who chose to participate in the study and those who did not, or who were unable to complete the study protocol $(\mathrm{p}=0.7)$. Global symptom relief of gut symptoms was reported by $8 / 14$ patients $(57 \% p=0.007$ figure 1$)$. A sub-group analysis demonstrated a significant reduction in both abdominal pain $(\mathrm{p}=0.001$ figure 2$)$ and distension $(\mathrm{p}=0.02$ figure 3$)$ respectively. There were no significant differences to anthropometric and biochemical features at follow up compared with baseline.

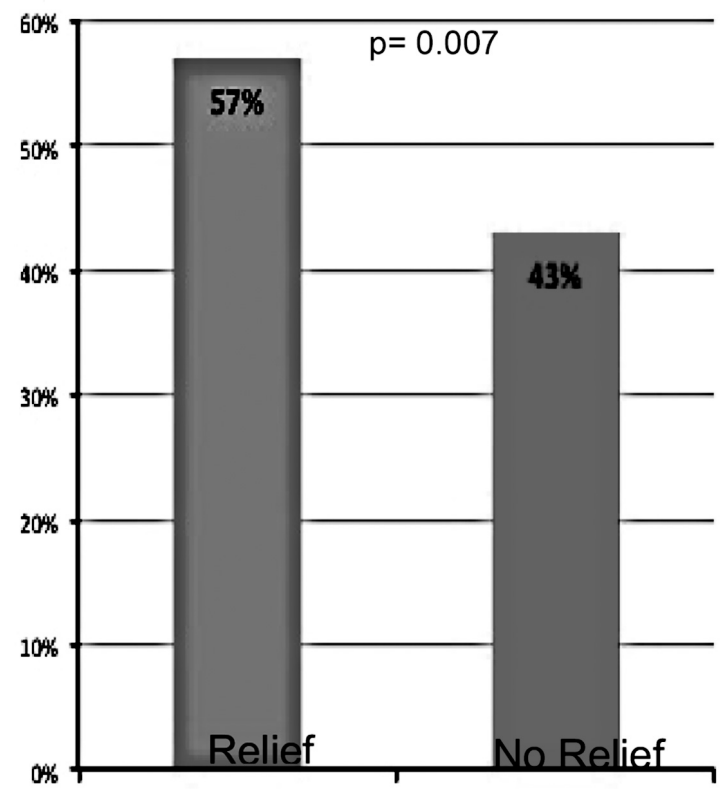

Abstract PWE-123 Figure 1 Adequate relief at follow up 


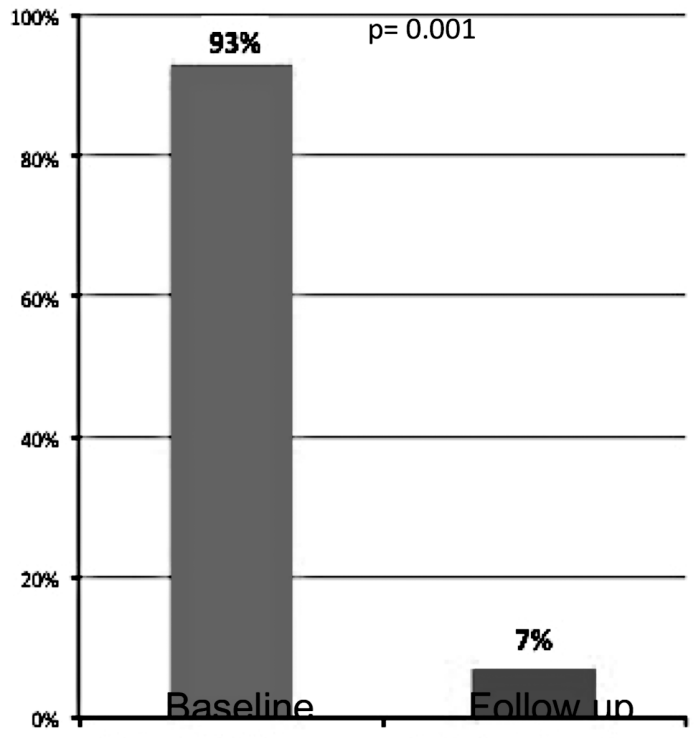

Abstract PWE-123 Figure 2 Abdominal pain present

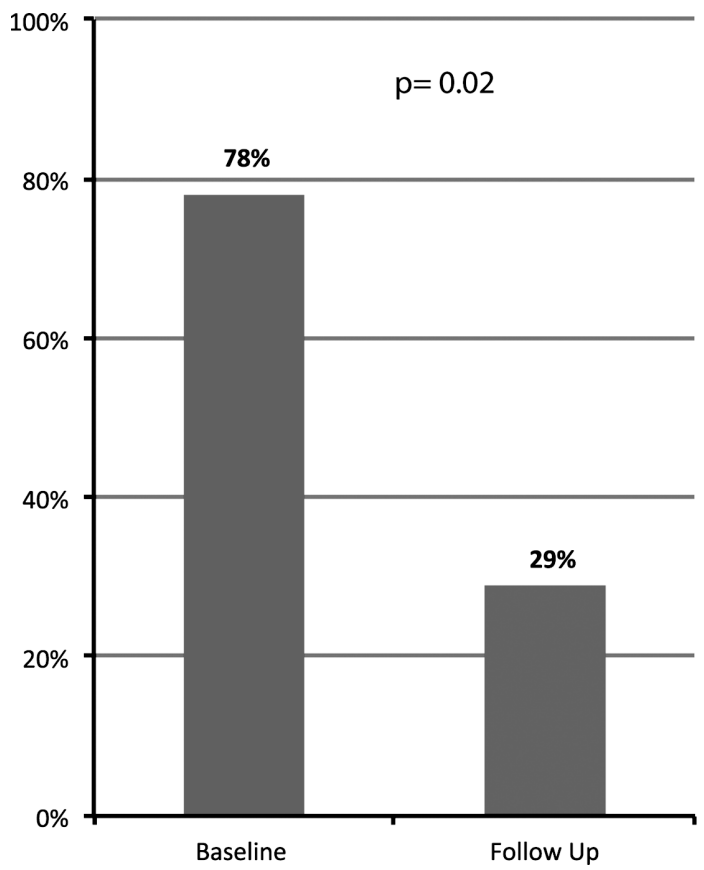

Abstract PWE-123 Figure 3 Abdominal distension present

Conclusion This is the first study to demonstrate that a LFD is an effective dietary treatment for patients with biopsy confirmed treated-CD and on going GI symptomology. Such patients should be seen by a specialist dietitian to improve adherence, ensure nutritional adequacy and appropriate reintroduction of FODMAP containing foods.

Disclosure of interest: None Declared

\section{PWE-124 HOW PATIENTS USE DIETARY INFORMATION FROM GP'S AND GASTROENTEROLOGISTS IN IBS SELF- MANAGEMENT}

Nick Trott ${ }^{*}$, Matthew Kurien, David Sanders. Department of Gastroenterology Royal Hallamshire Hospital, Sheffield, UK

\subsection{6/gutjnl-2018-BSGAbstracts.358}

Introduction In recent years there has been a renewed interest in dietitian-led nutritional treatments for Irritable Bowel Syndrome (IBS)-specifically the Low FODMAP (LFD) and Gluten Free Diets (GFD). Increases in diagnosis and a lack of suitably trained dietitians to deliver these modalities mean many patients only receive nutritional information from General Practitioners (GP's) and Gastroenterologists (GE's). Since the LFD and GFD are dietitian-led, the aim of this research was to qualitatively explore how people with IBS use and apply dietary information from GP's and GE's in IBS self management.

Methods An initially sample of 33 people $(7$ male) responded to a research request from the staff and student body of Sheffield University. 10 participants with a median age of 45 years (range 24-64, 2 male) matched the inclusion criteria-diagnosed with IBS (ROME IV) and used diet as their primary treatment. There were no differences in baseline demographics between patients who participated in the study and those who did not (gender $p=1.0$, age $p=0.9$ ). All participants had received dietary information from GP's and GE's for self managing their IBS symptoms; primarily advice on the LFD. Semistructured interviews were conducted (minimum an $r$ duration) and evaluated using Interpretive Phenomenological Analysis (IPA). IPA is a qualitative research method that employs phenomenological, and idiographic techniques to explore and explain participants lived experience. IPA is particularly suited to examine and understand how people with IBS make sense of the dietary information they are given and how this relates to the self-management of their symptoms.

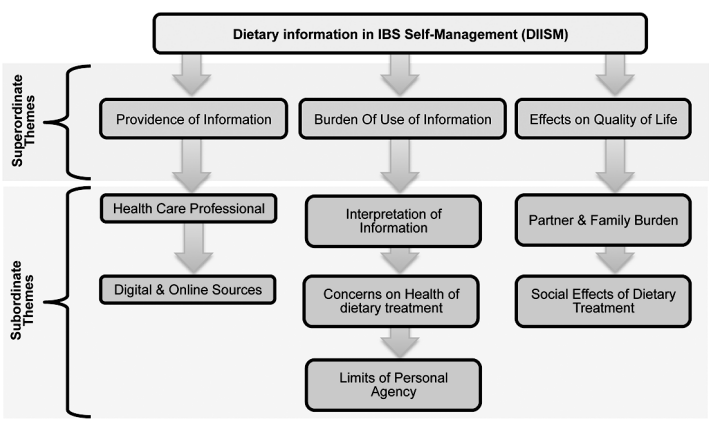

Abstract PWE-124 Figure 1 Dietary information in IBS self management(DIISM)

Findings The providence of nutritional information was important for the participants, information from GP's and GE's was valued as evidenced based. However, the information was

Abstract OTU-024 Table 1 Relative changes in colorectal cancer mortality (C18-21) by 5 year age band in England \& Wales from 2005 to 2016

\begin{tabular}{lccccccccc}
\hline & $45-49$ & $50-54$ & $55-59$ & $60-64$ & $65-69$ & $70-74$ & $75-80$ & $80-84$ & $85-89$ \\
\hline Men & $-18.0 \%$ & $-10.6 \%$ & $-23.1 \%$ & $-16.1 \%$ & $-22.7 \%$ & $-32.5 \%$ & $-19.5 \%$ & $-14.3 \%$ & $-8.8 \%$ \\
Women & $+14.7 \%$ & $-5.9 \%$ & $-0.7 \%$ & $-5.2 \%$ & $-24.1 \%$ & $-28.6 \%$ & $-10.9 \%$ & $-11.6 \%$ & $-2.9 \%$ \\
\hline
\end{tabular}


seen as very simplistic, often just 'food lists' with little or no personalisation to meet individual needs of the participants (figure 1). Digital online and resources were used to supplement the dietary information received form GP's and GE's, however this required additional interpretation and personalisation and led to negative effects on both the participants social and food-related quality of life.

Conclusion The participants found much of the nutritional information provide by GPS' and GE's to be overly generic and incomplete; in that it was difficult to apply in 'real life'. The findings in this study support the current clinical guidelines proposed by the both by NICE and the BDA that LFD and GFD's should still be considered second-line dietitian-led only interventions.

\section{PWE-125 EFFECT OF TURMERIC ON THE FAECAL VOLATILE ORGANIC METABOLITES IN HEALTHY INDIVIDUALS}

Gaurav Vashisht*, Kantida Koysombat, Rachael Hough, Lauren Lett, Sherry Browne, Naomi Lloyd, Chris Probert. University Of Liverpool, Liverpool, UK

\subsection{6/gutjnl-2018-BSGAbstracts.359}

Introduction Inflammatory bowel disease (IBD) affects the gut microbiome and metabolome. Curcumin, from turmeric, may be of benefit in some patients through action on the gut microbiome. Curcumin produces changes to bile acid secretion, and has a variety of direct effects on bacteria. Faeces release Faecal Volatile Organic Metabolites (VOMs), which partly reflect the gut microbiome. The aim of the study was to investigate the effects of turmeric on the VOMs in healthy individuals.

Methods 5 participants were enrolled in a before-during-after pilot study, in which they were asked to take a turmeric-free diet and then to consume $1.6 \mathrm{~g}$ of turmeric daily for 5 days. Faecal samples were collected at baseline, after 5 days of turmeric ingestion, and again 5 days after this, and frozen immediately. The samples were analysed by an investigator blinded using gas chromatography mass spectrometry. Analytes were identified using AMDIS software and compared using Metaboanalyst software: ANOVA, PCA, PLSADA, and Heatmap were employed.

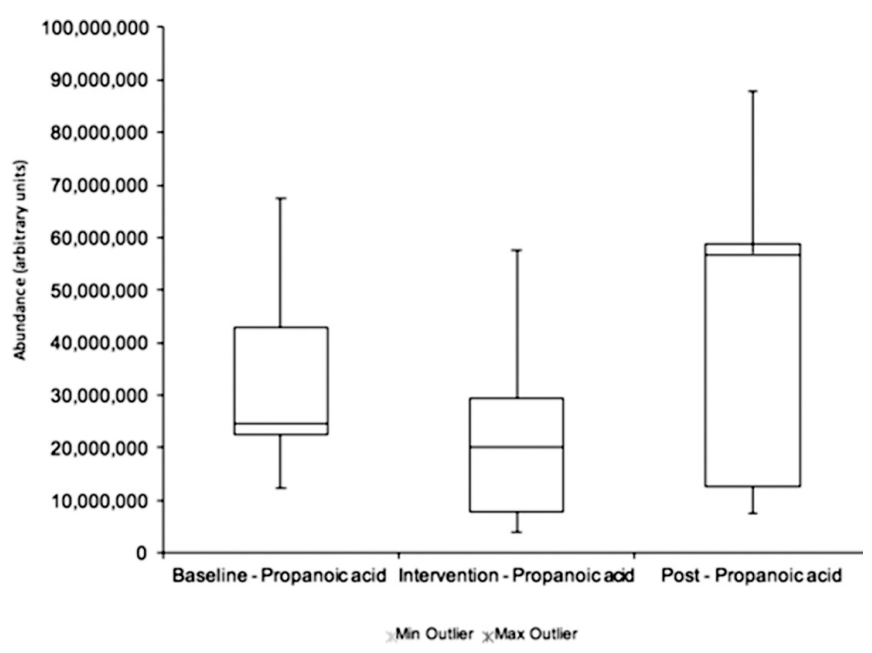

Abstract PWE-125 Figure 1 Box plot showing changes in abundance of propanoic acid amongst the comparison groups

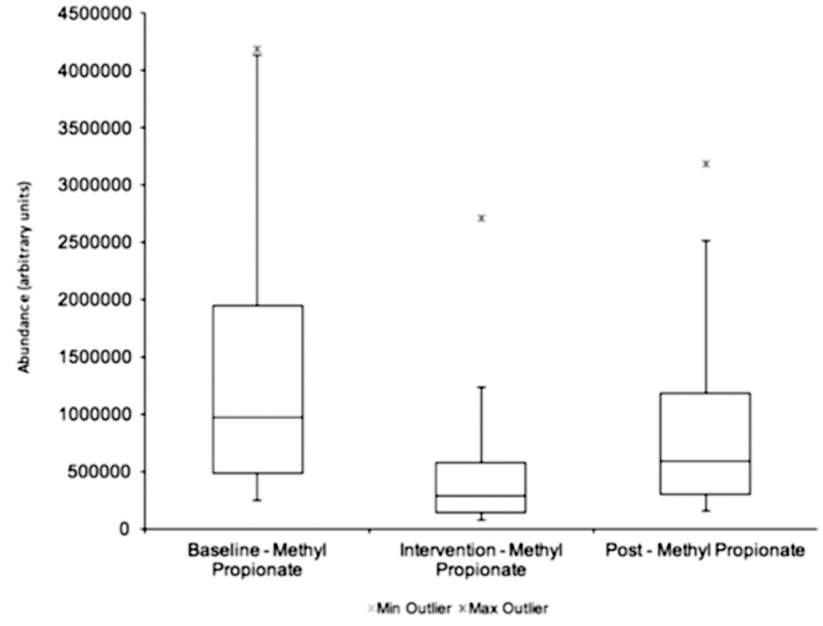

Abstract PWE-125 Figure 2 Box plot showing changes in abundance of methyl propionate amongst the comparison groups

Results ANOVA yielded 0 significant features. For most of the VOMs found between the comparison groups, $p>0.05$. Both PCA and PLSADA failed to show any separation by group. Heatmap analysis did not show any pattern in the abundance of VOMs. VIP scores showed a decrease in the abundance of propanoic acid and methyl propionate in the intervention samples when compared to baseline and post-turmeric consumption. The box plots created from raw data in Figure $1 \& 2$ demonstrated lower median abundances of intervention samples as compared to comparison groups. The study was underpowered to demonstrate significant change.

Conclusions This pilot study illustrates that two VOMs appears to become less abundant when turmeric is consumed: both appear related to propionibacteria metabolism. Studies in patients with IBD are warranted.

\section{PWE-126 LOW FODMAP DIET EFFECT ON IBS GASTROINTESTINAL MICROBIOME AND METABOLITES AND PREDICTION OF RESPONSE}

${ }^{1}$ Bridgette Wilson*, ${ }^{1}$ Megan Rossi, ${ }^{1}$ Tokuwa Kanno, ${ }^{2}$ Rachael Hough, ${ }^{2}$ Chris Probert, 1,3Peter Irving, ${ }^{1}$ A James Mason, ${ }^{1,3}$ Miranda Lomer, 'Kevin Whelan*. 'King's College London, London, UK; ${ }^{2}$ University of Liverpool, Liverpool, UK; ${ }^{3}$ Guy's and St Thomas' NHS Foundation Trust, London, UK

\subsection{6/gutjnl-2018-BSGAbstracts.360}

Introduction Prebiotic $\beta$-galactooligosaccharides (B-GOS) may counteract the microbiome modifying effect of the low FODMAP diet (LFD) in patients with irritable bowel syndrome (IBS). Faecal metabolites may predict why only some patients respond to the LFD paving the way towards more personalised treatment.

The aim of this randomised controlled trial (RCT) was to investigate: a) the impact of the LFD and LFD $+1.4 \mathrm{~g} / \mathrm{d}$ BGOS compared to Control on the gut microbiome in IBS, and b) if differences in faecal or urinary metabolites predict response to the LFD.

Methods A 3-arm RCT was performed in 69 IBS patients randomised to: Sham diet +Placebo (Control), LFD + Placebo (LFD) or LFD +B GOS. This study investigated global 